\title{
An Improved Z-Scheme for Overall Water Splitting Using Dye- Sensitized Calcium Niobate Nanosheets Synthesized by a Flux Method
}

Koya Hojo, ${ }^{1}$ Shunta Nishioka, ${ }^{2, \#}$ Yugo Miseki, ${ }^{3}$ Yoshinobu Kamakura, ${ }^{1,4}$ Takayoshi Oshima, ${ }^{1, \$}$ Kazuhiro Sayama, ${ }^{3}$ Thomas E. Mallouk, ${ }^{2,5}$ Kazuhiko Maeda*1

${ }^{1}$ Department of Chemistry, School of Science, Tokyo Institute of Technology, 2-12-1-NE-2 Ookayama, Meguroku, Tokyo 152-8550, Japan

${ }^{2}$ Department of Chemistry, University of Pennsylvania, 231 S. 34th Street Philadelphia, PA 19104, United States

${ }^{3}$ Global Zero Emission Research Center (GZR), National Institute of Advanced Industrial Science and Technology (AIST), West, 16-1, Onogawa, Tsukuba, Ibaraki, 305-8569, Japan

${ }^{4}$ Japan Society for the Promotion of Science, Kojimachi Business Center Building, 5-3-1 Kojimachi, Chiyoda-ku, Tokyo 102-0083, Japan

${ }^{5}$ International Center for Materials Nanoarchitectonics (WPI- MANA), National Institute for Materials Science (NIMS), Tsukuba, Ibaraki 305-0044, Japan

Present Addresses:

\# Department of Chemistry, School of Science, Tokyo Institute of Technology, 2-12-1-NE-2 Ookayama, Meguroku, Tokyo 152-8550, Japan

\$ Max Planck Institute for Solid State Research, Heisenbergstr. 1, Stuttgart 70569, Germany

*To whom corresponding authors should be addressed.

T.E.M.: mallouk@sas.upenn.edu; K.M.: maedak@chem.titech.ac.jp 
a)
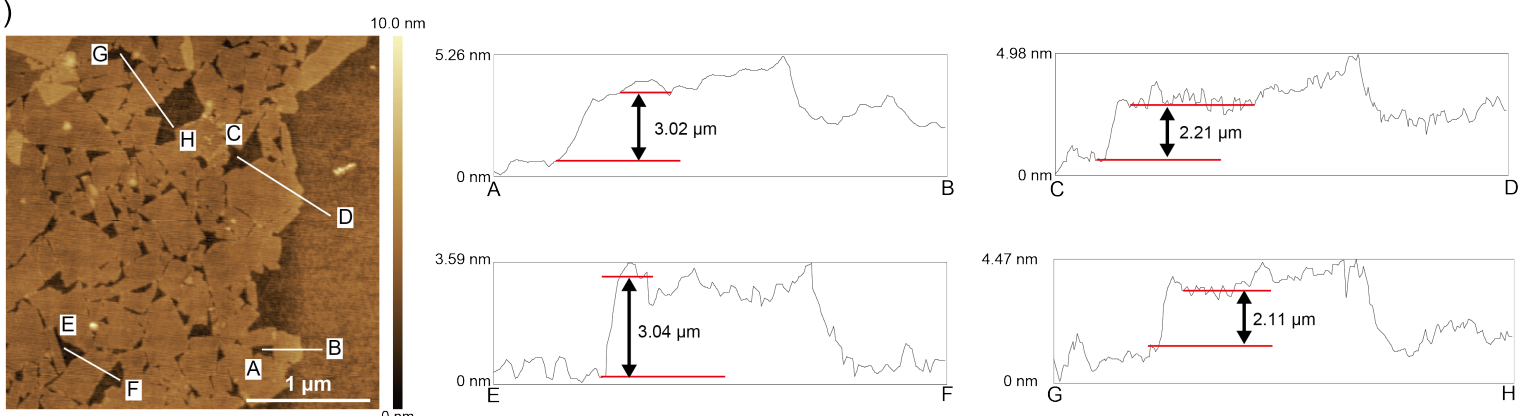

b)
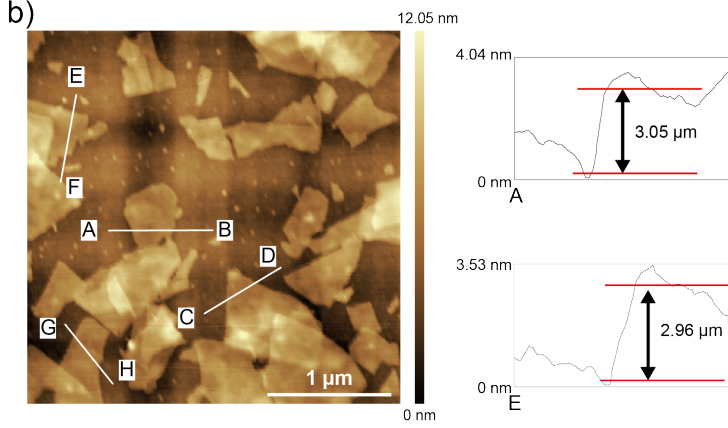

$4.24 \mathrm{~nm}$
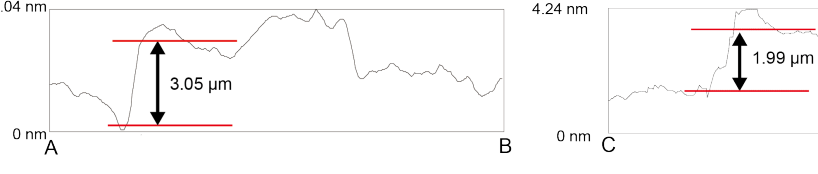

E

$3.78 \mathrm{~nm}$

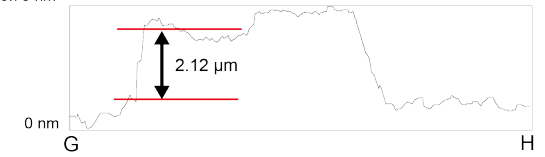

Figure S1. AFM images of TBA ${ }^{+}$-exfoliated $\mathrm{Ca}_{2} \mathrm{Nb}_{3} \mathrm{O}_{10} 0^{-}$prepared by the flux method and solid-state reaction, along with their height profiles.

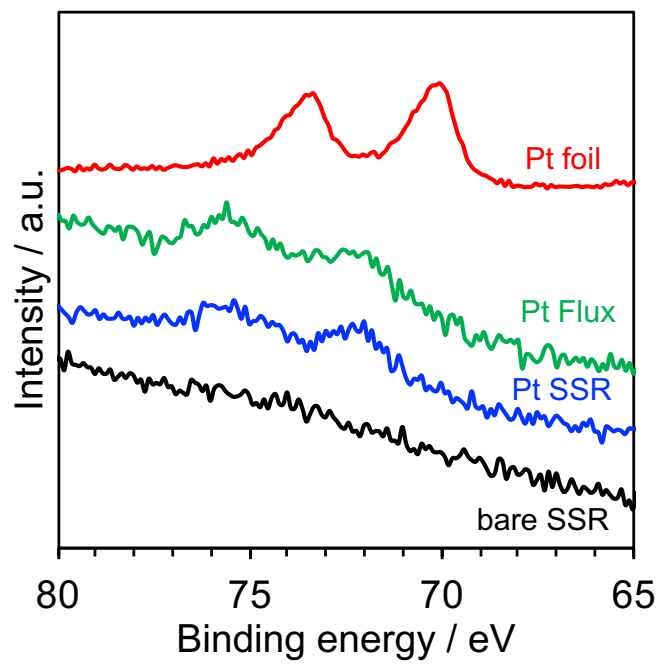

Figure S2. Pt-4f XPS spectra of $\mathrm{Pt} / \mathrm{HCa}_{2} \mathrm{Nb}_{3} \mathrm{O}_{10}$ nanosheets prepared by the flux method and solid-state reaction. Spectra of bare $\mathrm{HCa}_{2} \mathrm{Nb}_{3} \mathrm{O}_{10}$ synthesized by SSR and Pt foil were also shown as references. 
a) Flux

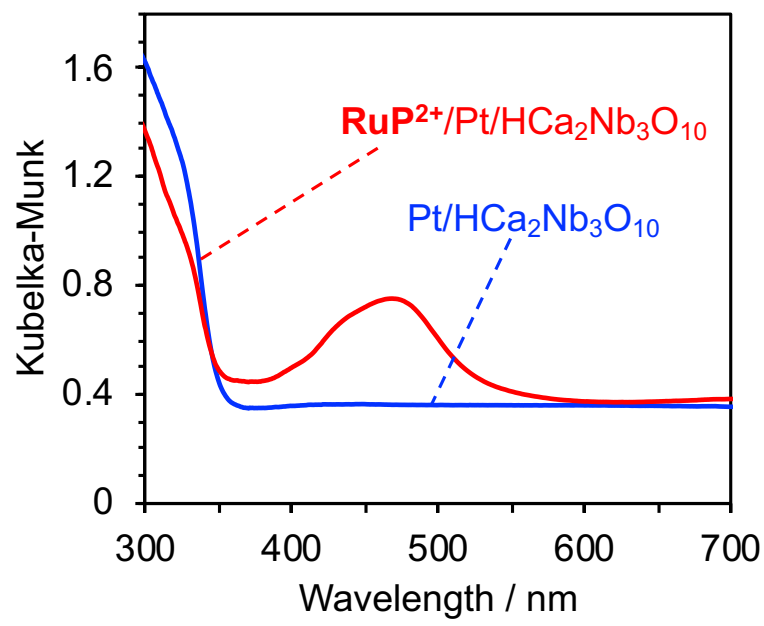

b) SSR

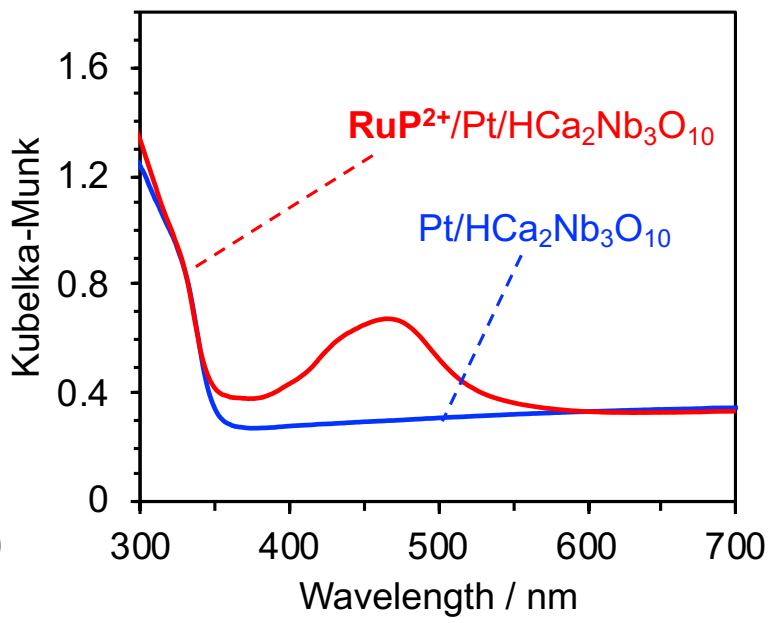

Figure S3. UV-visible DRS of $\mathrm{Pt} / \mathrm{HCa}_{2} \mathrm{Nb}_{3} \mathrm{O}_{10}$ nanosheets prepared by the flux method and solid-state reaction with and without adsorption of $\mathbf{R u P}^{2+}$.

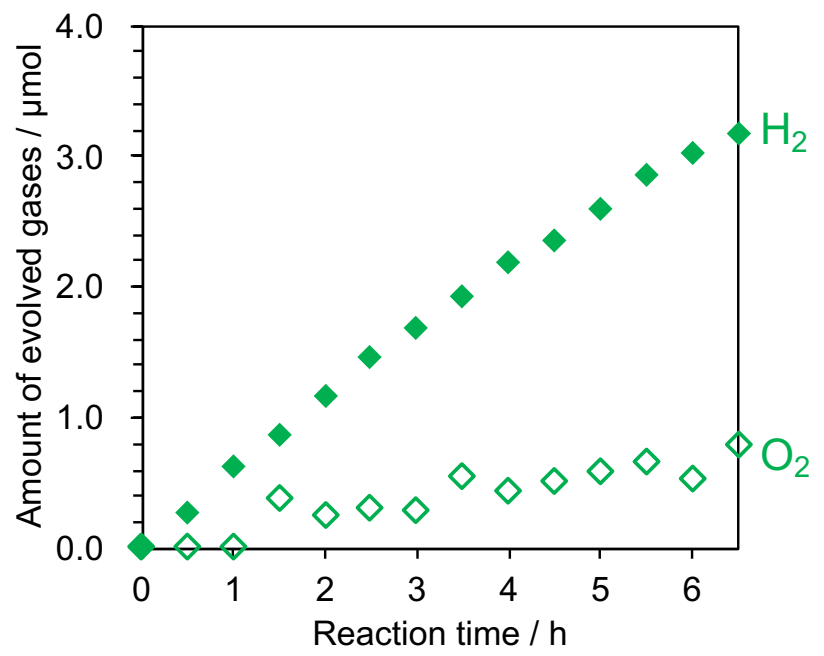

Figure S4. Time course of Z-scheme water splitting under artificial sunlight irradiation $\left(200 \mathrm{~mW} \mathrm{~cm}{ }^{-2}\right.$ = 2 Sun) over $\mathbf{R u P}{ }^{2+} / \mathrm{Pt} / \mathrm{HCa}_{2} \mathrm{Nb}_{3} \mathrm{O}_{10}$ nanosheets prepared by the flux method. Reaction conditions: $\mathbf{R u P}^{2+} / \mathrm{Pt} / \mathrm{HCa}_{2} \mathrm{Nb}_{3} \mathrm{O}_{10}$ nanosheets, $0.02 \mathrm{~g} ; \mathrm{PtO}_{x} / \mathrm{H}-\mathrm{Cs}-\mathrm{WO}_{3}, 0.05 \mathrm{~g}$; solution, aqueous NaI solution (5 $\mathrm{mM}, 100 \mathrm{~mL}, \mathrm{pH}=3.8-4.0)$. Solar-to-hydrogen conversion efficiency (STH) was calculated in the same manner as reported previously. ${ }^{\mathrm{S1}}$ 


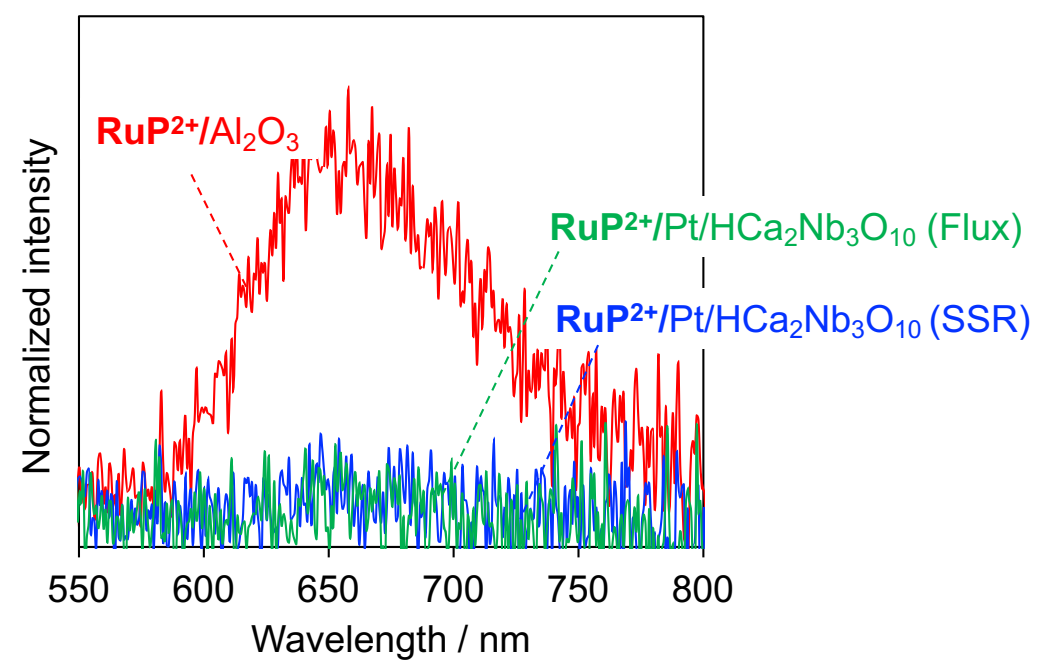

Figure S5. Steady-state emission spectra of $\mathbf{R u P}^{\mathbf{2}+} / \mathrm{Al}_{2} \mathrm{O}_{3}$ and $\mathbf{R u} \mathbf{P}^{\mathbf{2}+} / \mathrm{Pt} / \mathrm{HCa}_{2} \mathrm{Nb}_{3} \mathrm{O}_{10}$ nanosheets prepared by the flux method and solid-state reaction. Measurements were conducted by suspending the powder samples in Ar-saturated water under $460 \mathrm{~nm}$ excitation. The emission quantum yield of $\mathbf{R u P} \mathbf{P}^{2+} / \mathrm{Pt} / \mathrm{HCa}_{2} \mathrm{Nb}_{3} \mathrm{O}_{10}$ was found to be $0.1 \%$ in both synthesis methods, and that of $\mathbf{R u P} \mathbf{P}^{2+} / \mathrm{Al}_{2} \mathrm{O}_{3}$ was $4.2 \%$. The emission quantum yields of $\mathbf{R u} \mathbf{P}^{2+} / \mathrm{Pt} / \mathrm{HCa}_{2} \mathrm{Nb}_{3} \mathrm{O}_{10}$ synthesized by solid state reaction and $\mathbf{R u} \mathbf{P}^{2+} / \mathrm{Al}_{2} \mathrm{O}_{3}$ were similar to those reported in the previous report. ${ }^{\mathrm{S} 2}$

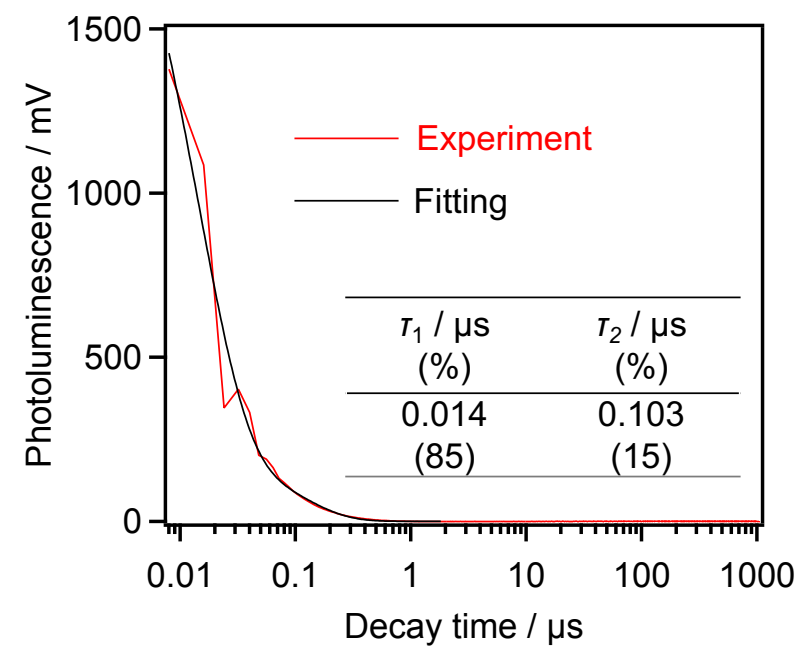

Figure S6. Time-dependent photoluminescence change in transient absorption measurement of $\mathbf{R u} \mathbf{P}^{2+}$ sensitized $\mathrm{HCa}_{2} \mathrm{Nb}_{3} \mathrm{O}_{10}$ nanosheets prepared by the flux method in aqueous $\mathrm{HCl}(\mathrm{pH}=4)$ monitored at $700 \mathrm{~nm}$. The inset table was fitted values of this decay curve by a double-exponential function. 


\section{Supporting Information (SI)}

\section{Fitting Analysis of Transient Absorption Spectroscopy}

To analyze the MLCT bleaching recovery of $\mathbf{R u P} \mathbf{P}^{2+}$ adsorbed on $\mathrm{HCa}_{2} \mathrm{Nb}_{3} \mathrm{O}_{10}$ nanosheets, we fit the decay curves by two-step procedures. The first step is a fitting of a photoluminescence signal that was recorded at $700 \mathrm{~nm}$ (see Figure S6 above) as a current of diode detector. The fitting was performed by using a double-exponential function (eq. S1).

$f(x)=y_{0}+A_{1} \exp \left(-\frac{x-x_{0}}{\tau_{1}}\right)+A_{2} \exp \left(-\frac{x-x_{0}}{\tau_{2}}\right)$,

Photoluminescence from dye molecules adsorbed on a solid surface is very complicated because it includes a lot of quenching processes, i.e. self-quenching of dyes, charge injection from excited states that are not thermally equilibrated and from thermalized MLCT excited state, in addition to light scattering. Moreover, the charge transfer kinetics from the lowest MLCT excited state can also be complex because some dye molecules are in different chemical environments than others. The convolution of the excited state decay with the timescale of laser excitation (the laser pulse duration is $1.85 \mathrm{~ns})$ makes the interpretation of the short lifetime components even more complicated. It is empirically known that, however, the early time data can be fitted by a double-exponential function, although there are many processes in such very short timescale, and it worked well also the fitting of photoluminescence signals in this study.

The second step of this analysis is a fitting of a MLCT bleaching recovery that was monitored at 475 $\mathrm{nm}$ (Figure 7) by a quintuple-exponential function (eq. S2) and by using the two lifetime values that obtained by the first step of this analysis. The two obtained values form eq. S1 were respectively substituted into $\tau_{1}$ and $\tau_{2}$ of eq. S2 and fixed when the second step fitting analysis was performed. In this

study, the finally obtained three lifetimes $\left(\tau_{3}, \tau_{4}\right.$ and $\left.\tau_{5}\right)$ were discussed. It is noted that the values of lifetimes derived from the first step $\left(\tau_{1}\right.$ and $\tau_{2}$ ) have low accuracy due to the low time resolution of these measurements $(8 \mathrm{~ns})$; nevertheless the values are worth to use for the second step fitting analysis because the photoluminescence signals were reproduced well.

$f(x)=y_{0}+\mathrm{A}_{1} \exp \left(-\frac{x-x_{0}}{\tau_{1}}\right)+\mathrm{A}_{2} \exp \left(-\frac{x-x_{0}}{\tau_{2}}\right)+\mathrm{A}_{3} \exp \left(-\frac{x-x_{0}}{\tau_{3}}\right)+\mathrm{A}_{4} \exp \left(-\frac{x-x_{0}}{\tau_{4}}\right)+$ $A_{5} \exp \left(-\frac{x-x_{0}}{\tau_{5}}\right)$ 


\section{Reference}

S1. Oshima, T.; Nishioka, S.; Kikuchi, Y.; Hirai, S.; Yanagisawa, K. I.; Eguchi, M.; Miseki, Y.; Yokoi, T.; Yui, T.; Kimoto, K.; Sayama, K.; Ishitani, O.; Mallouk, T. E.; Maeda, K. An Artificial Z-Scheme Constructed from Dye-Sensitized Metal Oxide Nanosheets for Visible Light-Driven Overall Water Splitting. J. Am. Chem. Soc. 2020, 142, 8412-8420.

S2. $\quad$ Nishioka, S.; Oshima, T.; Hirai, S.; Saito, D.; Hojo, K.; Mallouk, T. E.; Maeda, K. Excited Carrier Dynamics in a Dye-Sensitized Niobate Nanosheet Photocatalyst for Visible-Light Hydrogen Evolution. ACS Catal. 2021, 11, 659-669. 